Provided for non-commercial research and education use. Not for reproduction, distribution or commercial use.

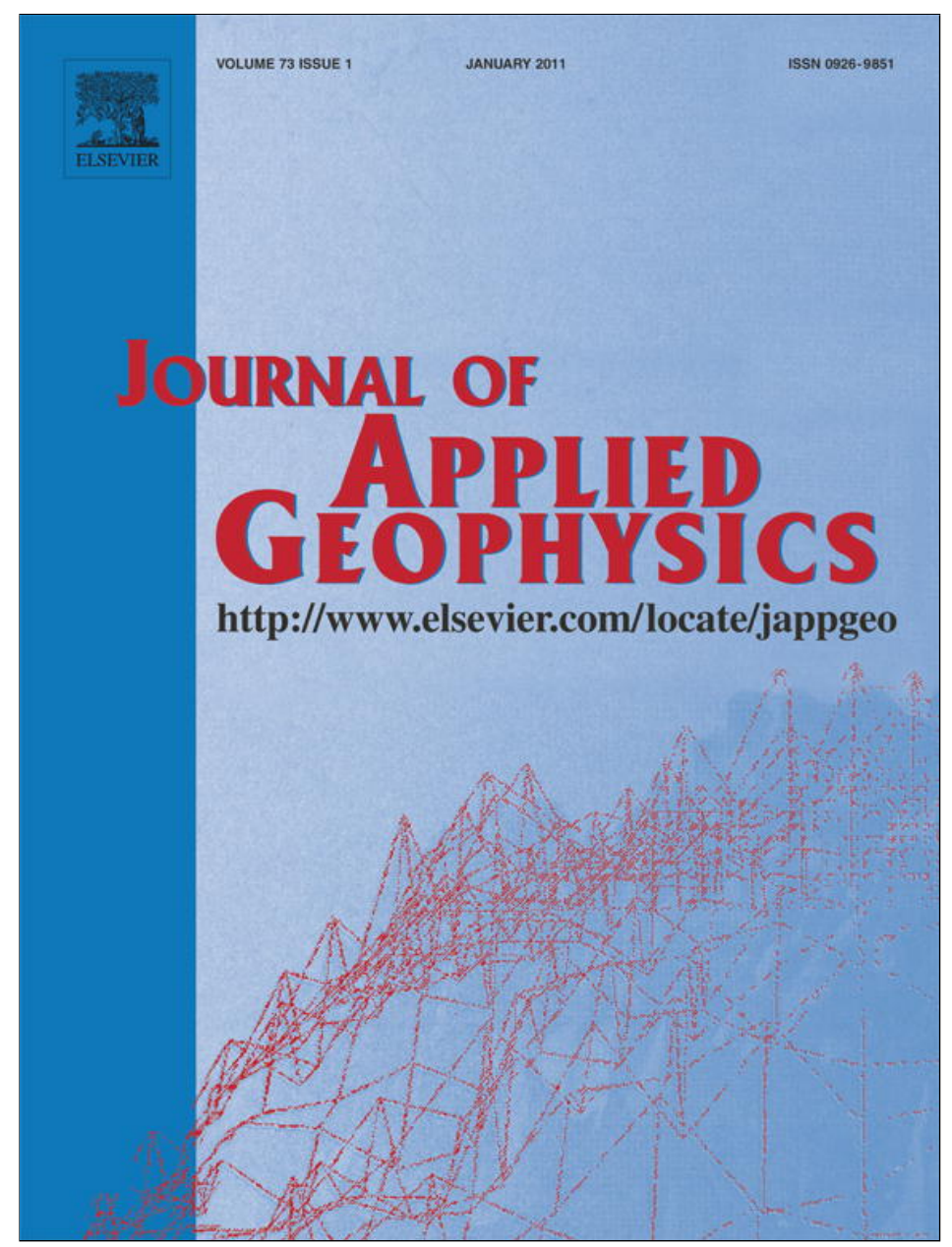

(This is a sample cover image for this issue. The actual cover is not yet available at this time.)

This article appeared in a journal published by Elsevier. The attached copy is furnished to the author for internal non-commercial research and education use, including for instruction at the authors institution and sharing with colleagues.

Other uses, including reproduction and distribution, or selling or licensing copies, or posting to personal, institutional or third party websites are prohibited.

In most cases authors are permitted to post their version of the article (e.g. in Word or Tex form) to their personal website or institutional repository. Authors requiring further information regarding Elsevier's archiving and manuscript policies are encouraged to visit:

http://www.elsevier.com/copyright 


\title{
Expanding the possibilities of two-dimensional multielectrode systems, with consideration to earlier geoelectric arrays
}

\author{
Sándor Szalai, László Szarka* \\ Geodetic and Geophysical Research Institute of the Hungarian Academy of Sciences, H-9401 Sopron, POB 5, Hungary
}

\section{A R T I C L E I N F O}

\section{Article history:}

Received 18 June 2010

Accepted 10 June 2011

Available online $\mathrm{xxxx}$

\section{Keywords:}

Geoelectric array

Multielectrode measurements

Two-dimensional

Electrode configuration

\begin{abstract}
A B S T R A C T
Recent two-dimensional multielectrode measurements are restricted to only a few geoelectric arrays. Realizing that specific features of nearly 90 other arrays are totally ignored, all original intentions as published about the development of new geoelectric arrays were reviewed. Apart from arrays, either already applied in two-dimensional geoelectric arrays or impossible to be applied in such systems, 61 forgotten once-developed arrays were found. These provide altogether 102 various solutions, which would be able to increase the efficiency of two-dimensional multielectrode measurements in some respect. 46 array solutions are able to enhance the depth of investigation; 9/11 array solutions give better vertical/horizontal resolution; 17 array solutions provide better planview images; 8 array solutions are worth applying in areas with limited access; 11 array solutions may reduce the effect of near-surface inhomogeneities. By reviving these forgotten arrays, it will be possible to develop versatile multielectrode systems, which are more adaptive to the diverse field needs.
\end{abstract}

(c) 2011 Elsevier B.V. All rights reserved.

\section{Introduction}

Due to the advent of multielectrode arrays and effective inversion tools, geoelectrics has witnessed a rapid development in the last decades, but several old questions have not been answered yet. One of those questions is which array performs the best for a given field problem. In spite of the fact that there are about one hundred independent geoelectric arrays (Szalai and Szarka, 2008a), nearly 90\% of field measurements are carried out with one of the following five arrays: Schlumberger, Wenner- $\alpha$, Wenner- $\beta$, dipole-dipole and poledipole. These are shown in Fig. 1, together with the number of publications about various arrays. All these arrays are collinear, and, with the exception of the pole-dipole (P-Dp) array, four-electrode.

There exist only a few non-conventional multielectrode array schemes and packages, which aim at an optimization of geoelectric imaging of the subsurface [e.g., (Furman et al., 2004; Stummer et al., 2004; Wilkinson et al., 2006; Xu and Noel, 1993)]. All these efforts were reduced to the application of various four-electrode arrays. The only three-electrode array, which has been used for the optimization of 2D (two-dimensional) multielectrode measurements, is the poledipole (P-Dp) array (Candansayar are Başokur, 2001).

It should not be forgotten that the remaining nearly ninety arrays are also issues of certain intentions. Nowadays, due to the over-

\footnotetext{
* Corresponding author. Tel.: + 3699 508342; fax: + 3699508355.

E-mail addresses: szalai@ggki.hu (S. Szalai), szarka@ggki.hu (L. Szarka).
}

domination of the aforementioned few array types of 2D multielectrode arrays, many once-known features of these approximately ninety arrays are nearly totally forgotten. Therefore, it was decided to collect the original intentions as published, together with the suggested array solutions; then those arrays were selected, which could easily be built into 2D multielectrode systems.

The starting point for the present study is the authors' compilation of all-published geoelectric arrays (Szalai and Szarka, 2008a). In that paper, $92(+10)$ independent geoelectric arrays were collected and classified into eight classes. Array figures, array numbers and all notations in this paper follow those in (Szalai and Szarka, 2008a). The classification of arrays in (Szalai and Szarka, 2008a) is based on three divalent parameters closely related to the components of the definition of the term "geoelectric array": a) superposition: if the number of potential difference measurements is more than one, the array is said to be "superposed", otherwise the array is "nonsuperposed"; b) focusing: if more than one current circuit is applied, the array is said to be "focused", otherwise the array is "non-focused"; c) collinearity: if the alignment of the electrodes is linear, and array is said to be "collinear", otherwise the array is "non-collinear".

In this way, there are altogether $2^{3}=8$ groups, comprising altogether 92 independent geoelectric arrays. The eight classes are as follows:

I. 22 so-called "simplest" (non-superposed, non-focused, collinear) arrays,

II. 12 "simple non-collinear" (non-superposed, non-focused, noncollinear) arrays, 
The potential difference is measured: 1801 (98\%)
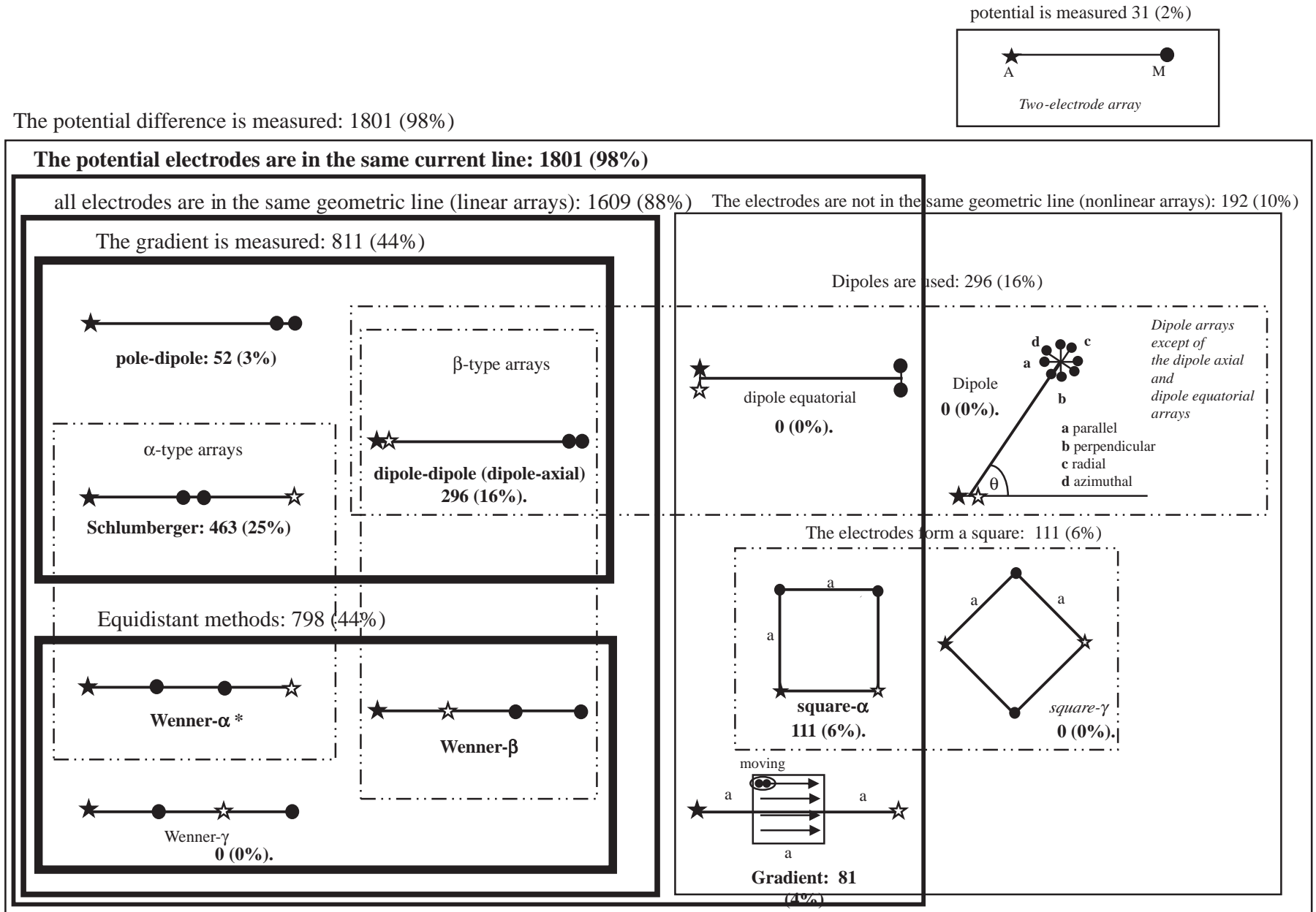

Fig. 1. Internet references to geoelectric arrays (according to Google, November 2009).

III. 10 "simple focused" (non-superposed, focused, collinear) arrays,

IV. 19 "simple superposed" (superposed, non-focused, collinear) arrays,

V. 6 non-superposed, focused, non-collinear arrays,

VI. 22 superposed, non-focused, non-collinear arrays,
VII. 1 superposed, focused, collinear array,

VIII. Superposed, focused, non-collinear arrays, which are still unrealized.

Only ten further published arrays (the so-called "composite" arrays) do not fit into either of these classes.

Table 1

Original intentions, suggested array solution techniques, and the number of arrays solutions, classified into four main considerations.

\begin{tabular}{|c|c|c|c|c|}
\hline \multirow{2}{*}{$\begin{array}{l}\text { Main considerations } \\
\text { Simplicity (1) }\end{array}$} & \multirow{2}{*}{$\begin{array}{l}\text { Original intentions } \\
\text { Increasing the measuring signal }\end{array}$} & \multirow{2}{*}{$\begin{array}{l}\text { Suggested solution techniques } \\
\text { C approaches } \mathrm{P}-(1 \mathrm{a})\end{array}$} & \multicolumn{2}{|c|}{$\begin{array}{l}\text { Number of } \\
\text { array solutions }\end{array}$} \\
\hline & & & 9 & 61 \\
\hline & Reducing the number of moving electrodes & $\mathrm{C}$ and/or $\mathrm{P}$ is at infinity $-(1 \mathrm{~b})$ & 21 & \\
\hline & Reducing the total length of cables & Dipole - (1c) & 13 & \\
\hline & Mapping without moving all electrodes & Some of the electrodes are fixed - (1d) & 10 & \\
\hline & Measurement in areas with limited access & Asymmetrical - (1e) & 8 & \\
\hline \multirow[t]{5}{*}{ Anomaly purification (2) } & Direct observation of the "pure" anomaly & Null $-(2 a)$ & 25 & 36 \\
\hline & Elimination of near-surface effects & Offset $-(2 b)$ & 2 & \\
\hline & & Difference - (2c) & 2 & \\
\hline & & Two-sided - (2d) & 5 & \\
\hline & & Change the identity of the electrodes $-(2 \mathrm{e})$ & 2 & \\
\hline \multirow[t]{7}{*}{ Studying inhomogeneity, anisotropy (3) } & Measuring resistivity changes in $x$ & Lee type $-(3 a)$ & 3 & 54 \\
\hline & (measuring) direction & Derivation - (3b) & 12 & \\
\hline & Measuring resistivity changes in $y$ direction & Vector - (3c) & 5 & \\
\hline & & Tensor $-(\mathbf{3 d})$ & 5 & \\
\hline & & Further nonlinear arrays - (3e) & 16 & \\
\hline & Measuring resistivity changes in $z$ & Mini-sounding - (3f) & 11 & \\
\hline & (vertical) direction & Differential depth - (3g) & 2 & \\
\hline Depth of investigation enhancement (4) & Sending the currents deeper & Focusing $-(4)$ & 17 & 17 \\
\hline
\end{tabular}




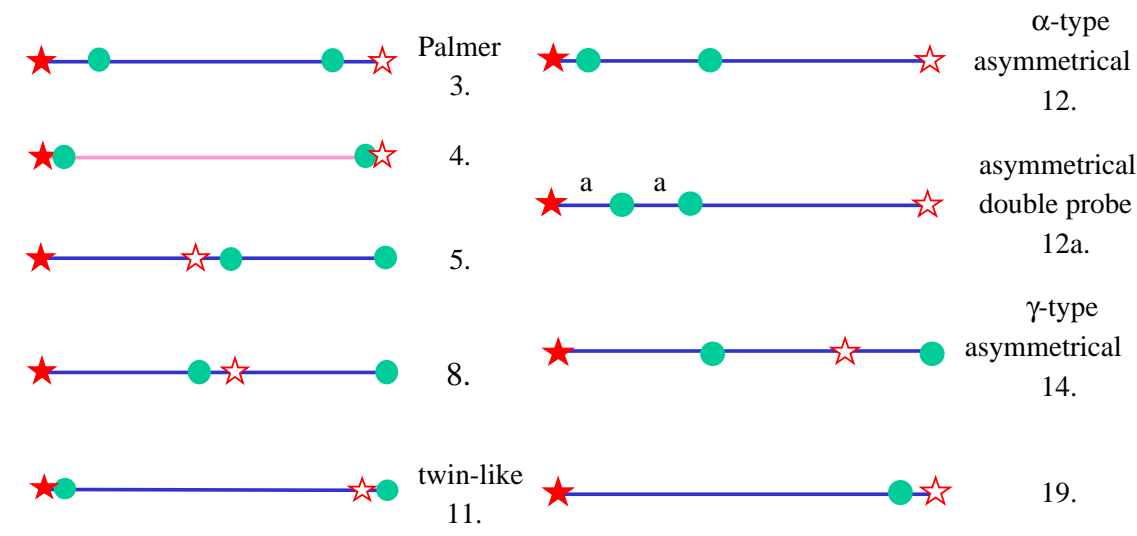

Fig. 2. The nine electrode arrays, which represent solution technique 1a ("C approaches P") to satisfy the original intention "Increasing the measuring signal" with the main consideration "Simplicity". The electrode arrays are identified by their number (listed in (Szalai and Szarka, 2008a)) and name (if any). C: current (source or sink electrode) Source/sink electrodes are full/empty stars. P: potential electrode (full circles) in all figures.

\section{Original intentions and the suggested array solutions}

The large variety of field situations has resulted in a large number of surface geoelectric arrays. Table 1 shows the list of original intentions, which have emerged so far, and the suggested solution techniques. In geophysical literature 11 such original intentions and altogether 18 solution techniques we found, which could be classified around four main considerations, namely: (1) simplicity, (2) anomaly purification, (3) local inhomogeneity and anisotropy, and (4) sending the currents more vertically into the ground. Some of the arrays represent multiple solutions, i.e. they might be issues with slightly different intentions. This is the reason that the total number of solution techniques in Table 1 (168) is higher than the total number of independent arrays (92).

As shown in Table 1, the arrays with light gray background have already been used in 2D multielectrode measurements; the arrays with a dark gray background are impossible to have built into 2D multielectrode measurements.

\subsection{Simplicity (solution technique 1)}

The main consideration, "simplicity", appeared in five different original intentions and in altogether 61 arrays, as listed in Table 1. Fig. 2 shows only those arrays from the collection in (Szalai and Szarka, 2008a), in which the C-P distance is very small compared to the array length. These nine solution techniques form group 1a. By applying these arrays, the vertical resolution is increased (Szalai et al., 2009), but the increased vertical resolution is accompanied by a decrease in the depth of investigation. The nine arrays in group 1a are useful in multielectrode systems only in the case of a large number of electrodes, otherwise the number of measuring points would be very small.

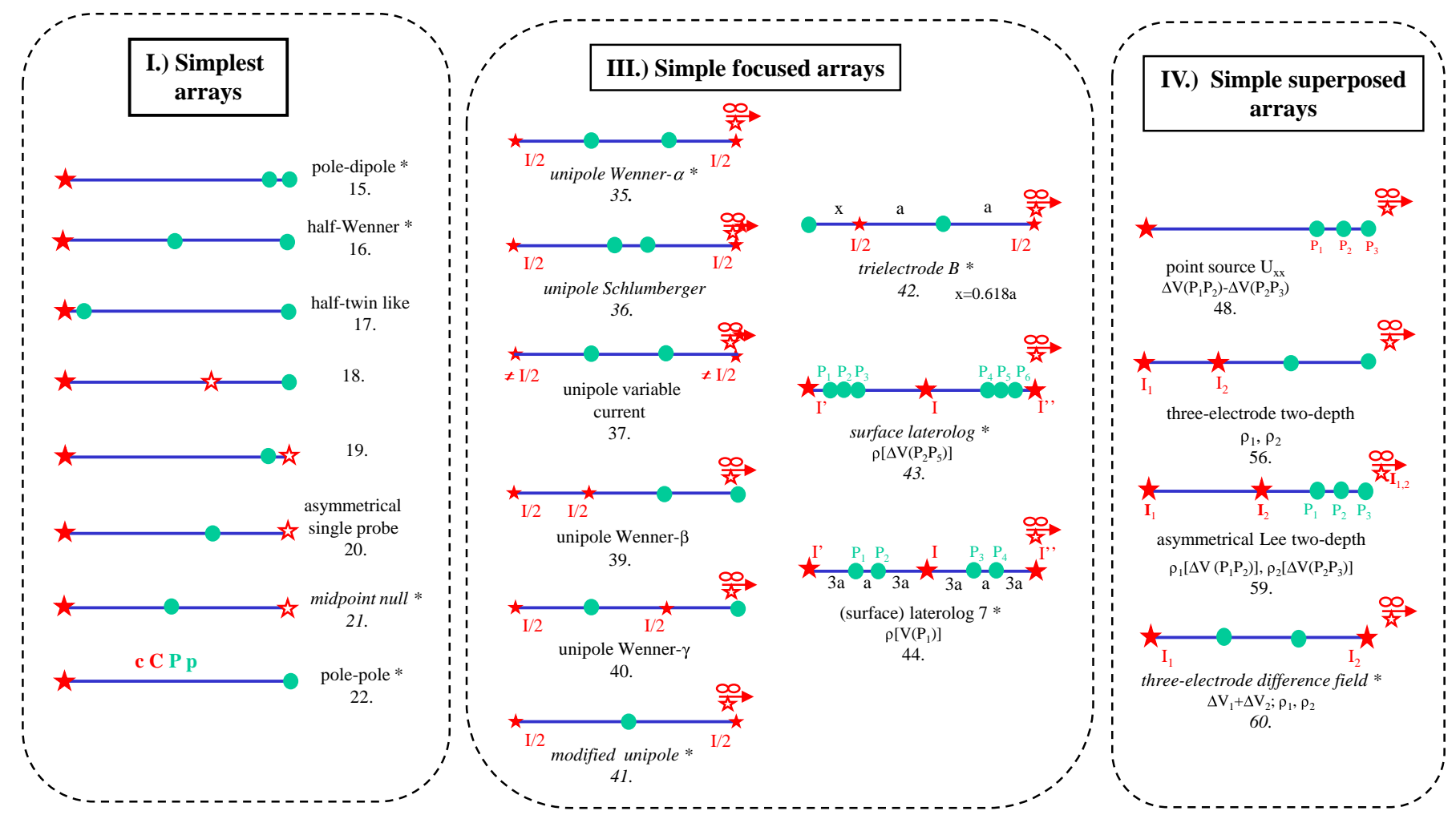

Fig. 3. The 21 electrode arrays, which represent solution technique $1 b$ ("C and/or P is at infinity") to satisfy the original intention "Reducing the number of moving electrodes" with the main consideration "Simplicity". The names of array groups as used in (Szalai and Szarka, 2008a) are shown in rectangles with dashed lines. 


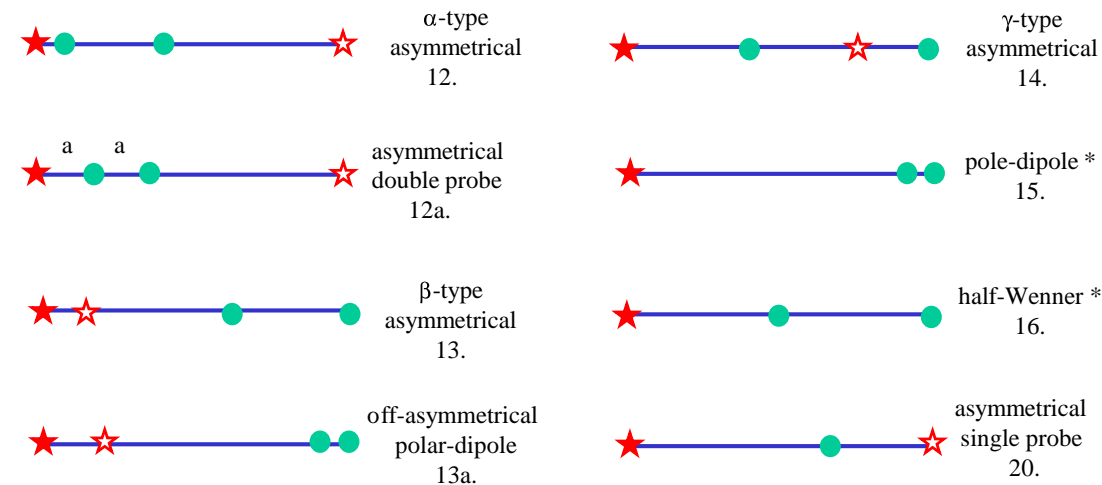

Fig. 4. The eight electrode arrays, which represent solution technique 1e ("Asymmetrical") to satisfy the original intention "measurement in areas with limited access" with the main consideration "Simplicity".

Arrays having electrode(s) at infinity (solution technique 1b: " $\mathrm{C}$ and/or P are/is at infinity") are much less frequently used in multielectrode systems than arrays without electrodes at infinity. Their depth of investigation is larger than that of a significant part of the four-electrode systems, but their field use is more complicated than that of four-electrode arrays (Candansayar and Başokur, 2001). There are altogether 21 such arrays, shown in Fig. 3.

The array solutions in group 1c ("Dipole"), due to the high horizontal resolution power of the arrays, are already permanent constituents of multielectrode systems. Stummer et al.(2004) also
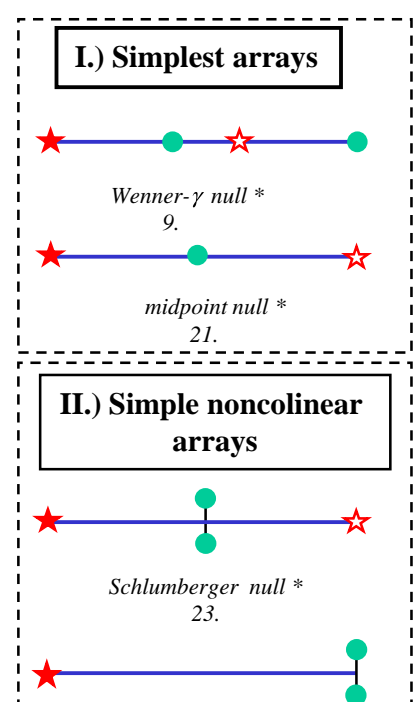

three-electrode null *

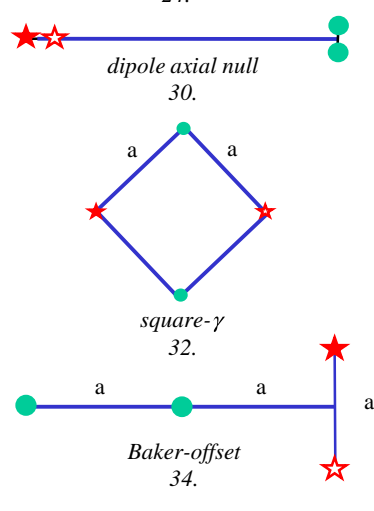

\section{III.) Simple focused arrays}
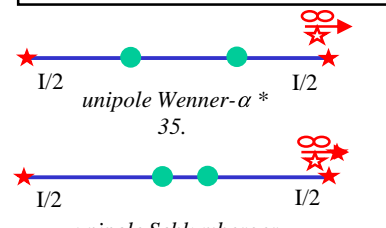

unipole Schlumberger

36.
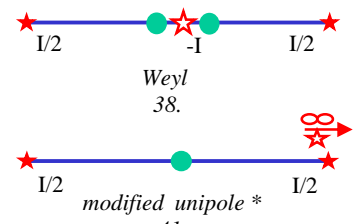

41.

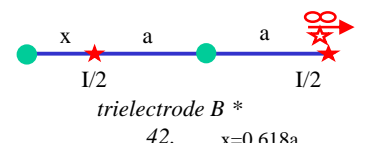

42. $\mathrm{x}=0.618 \mathrm{a}$

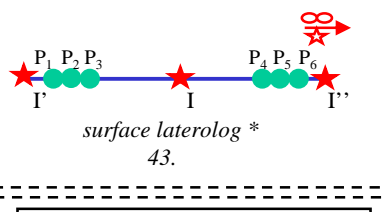

IV.) Simple superposed arrays

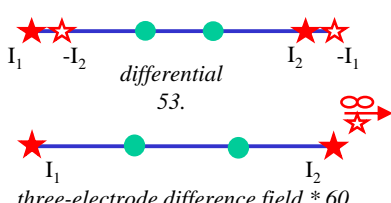

V.) Focused noncolinear arrays

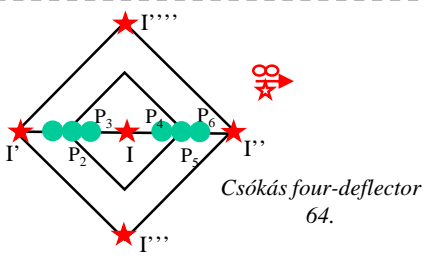

VI. Superposed noncolinear arrays
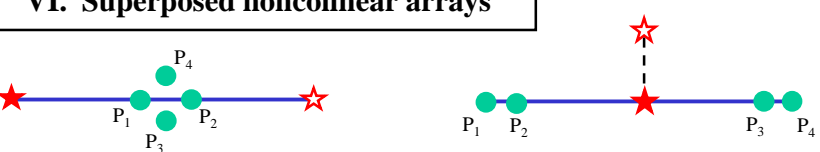

orthogonal 1

82.

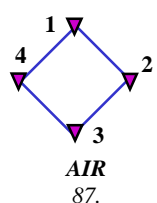

$\begin{array}{llll}1 & 2 & 3 & 4\end{array}$

I. C P P C

II. C C P P

III. C P C P
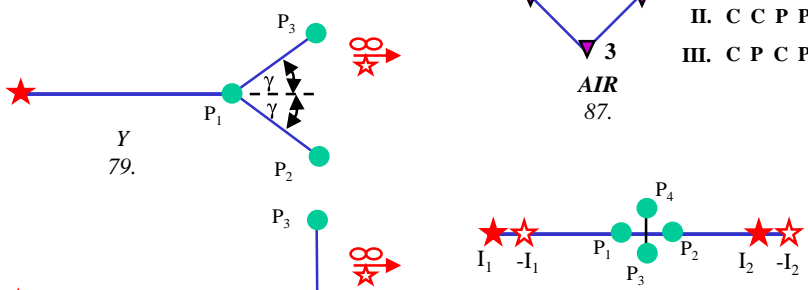

two-sided dipole axial superposed null *

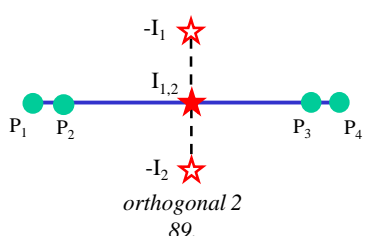

89.

Fig. 5. The 25 electrode arrays, which represent solution technique 2a ("Null arrays") to satisfy the original intention "direct observation of the pure anomaly" with the main consideration "Anomaly purification". Stars indicate alternative names (see them in (Szalai and Szarka, 2008a). Null arrays are typed in cursive. The names of array groups as used in Szalai and Szarka(2008a) are shown in rectangles with dashed lines. 
emphasized their basic role in multielectrode measurements: in the array set by Stummer et al.(2004), the first nearly 600 arrays are nonstandard bipole-bipole arrays. Arrays in group 1d ("Some of the electrodes are fixed") have also been built into multielectrode systems.

Where multielectrode system cannot be easily deployed (e.g. in built-up areas), the "asymmetrical" arrays might be useful (see solution technique 1e). A joint application of various AMN, MNB variants of the pole-dipole array give information from a wider area (Candansayar and Başokur, 2001). There are eight such arrays, shown in Fig. 4.

\subsection{Anomaly purification (solution technique 2)}

The objective of "direct observation of the pure anomaly" should be discussed in details, since it has altogether 25 elements. About one fourth of all known arrays belong to this group, as shown in Fig. 5. These arrays are called "null arrays" (Szalai et al., 2002), since their response over a homogeneous half-space is "null" (zero). Null arrays are not applied routinely. The first null array realizations were quite complicated: either they were focused arrays (requiring at least two current circuits, see classes III and V in Fig. 5), or superposed arrays, which require at least two potential difference measurements (groups IV and VI in Fig. 5). As it was demonstrated by Szalai et al.(2002), it is possible to construct null arrays in much simpler ways, namely by a suitable positioning of the electrodes (group II in Fig. 5). Depth of investigation values are, at least under some special conditions, higher than by using traditional arrays (Roy and Apparao, 1971; Szalai and Szarka, 2008b). Collinear arrays (group I in Fig. 5) can also be constructed by the "null" concept. The most promising among those null arrays for 2D multielectrode measurements, both from a practical and a theoretical point of view, is the three-electrode midpoint null (or MAN) array (Szalai et al., 2005), where one of the current electrodes is at halfway of the distance between the two potential electrodes. The "null" concept has a real, but not-yet exploited potential in multielectrode systems.

Further solution techniques corresponding to the objective of eliminating near-surface effects (suggested array solutions $2 b, c, d$
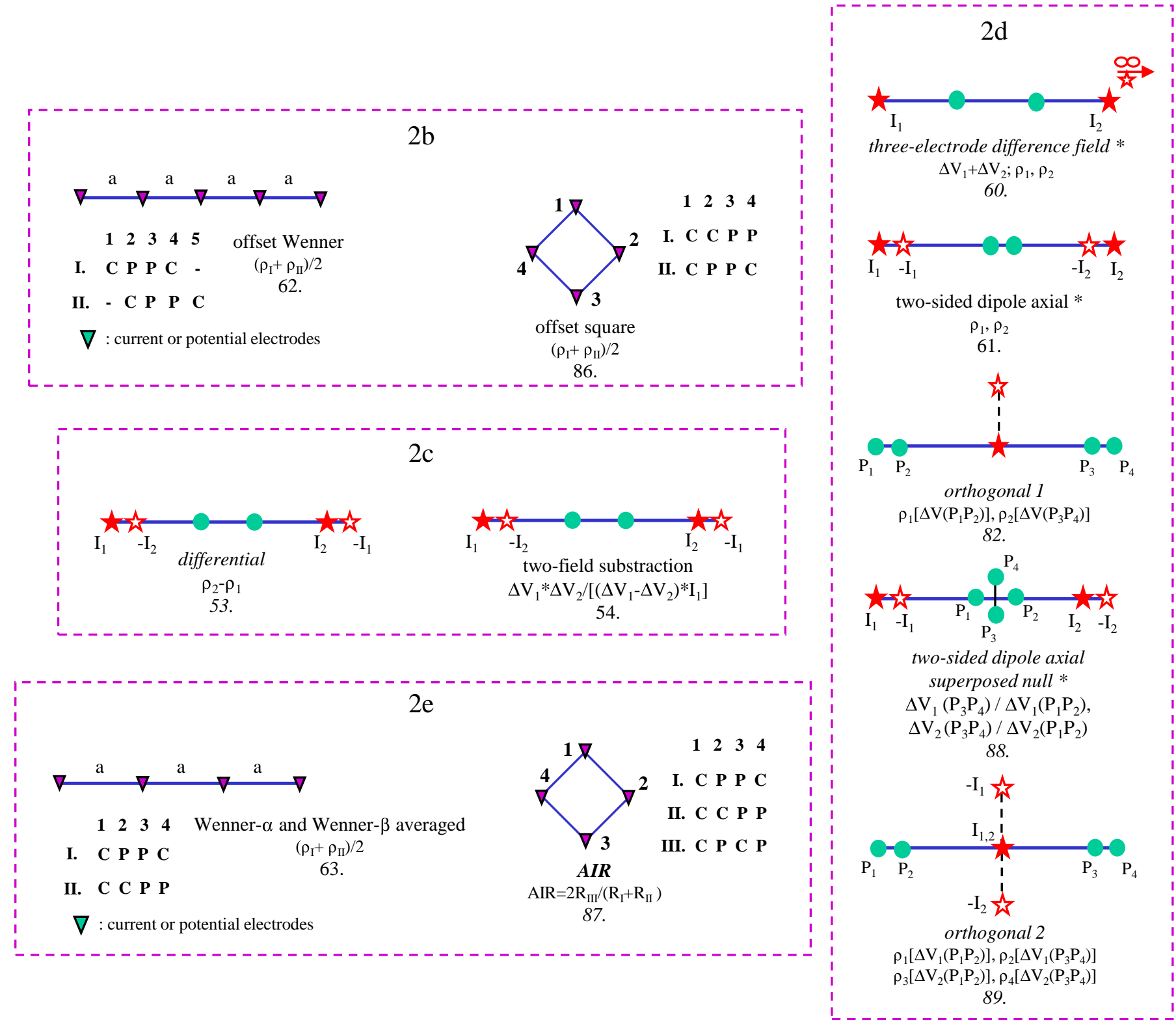

Fig. 6. The 11 electrode arrays, which represent solution techniques $2 \mathrm{~b}, \mathrm{c}, \mathrm{d}$, and e ("Offset", "Difference”, "Two-sided”, “Electrode identity changing”) to satisfy the original intention "elimination of near-surface effects" with the main consideration "Anomaly purification". 
and e, i.e. "Offset", "Difference", "Two-sided", "Electrode identity changing") are shown in Fig. 6. All linear arrays among them could be directly built into multielectrode systems, although with modified data processing.

\subsection{Studying local inhomogeneity, anisotropy (solution technique 3)}

Local inhomogeneity and/or local anisotropy (see main consideration no. 3 in Table 1) is determined via studying resistivity changes in the $x, y, z$ directions, where $x$ is the profile direction $z$ is pointed downward. While the solution technique 3a ("Lee-type": not shown, see the dark gray background in Table 1) seems unrealistic from the point of view of 2D multielectrode systems, the solution technique $3 \mathrm{~b}$ ("Derivation") is promising (see Fig. 7). In Fig. 7, instead of showing only the collinear arrays, which are compatible to 2D multielectrode systems, all those arrays are presented, which provide either the first or the second derivative of the electric potential. It should be noted that a large part of these arrays were published at first by Sapuzhak (1967). With one single exception, all arrays, which provide either the first or the second derivative according to $x$ are based on four arrays, and are used in the field (and indicated with gray background). These are the four arrays: Wenner- $\alpha$, Wenner- $\beta$ ( $\beta$-type dipole and $\beta$-type bipole) and half-Wenner- $\beta$. These arrays were also listed in group 1a (see Fig. 2), so they have the same advantages and disadvantages as listed there. With the exception of the dipole axial array, the array, which measures the second derivative has only been applied by Mousatov et al.(2002). It is probable that these arrays have promise both in multielectrode and traditional arrays.

Solution techniques 3c, d, and e ("Vector", "Tensor" and "Further nonlinear arrays", see Table 1) are not suitable for 2D multielectrode systems, since all of them are non-collinear arrays. At the same time, they might be useful in 3D arrays.

Solution techniques $3 \mathrm{f}$ and $3 \mathrm{~g}$ ("Mini-sounding" and "Differential depth", see Table 1) are collinear, and they have already been automatically built into multielectrode systems. In Table 1 both solution techniques are shown with a light gray background.

\subsection{Sending the currents more vertically into the ground (solution} technique 4)

The so-called "focused" arrays (solution technique 4, see in Fig. 8) were intended to send the currents deeper into the ground than it is possible by using other arrays. By using focused arrays, a larger depth of investigation was expected than with other arrays, but the

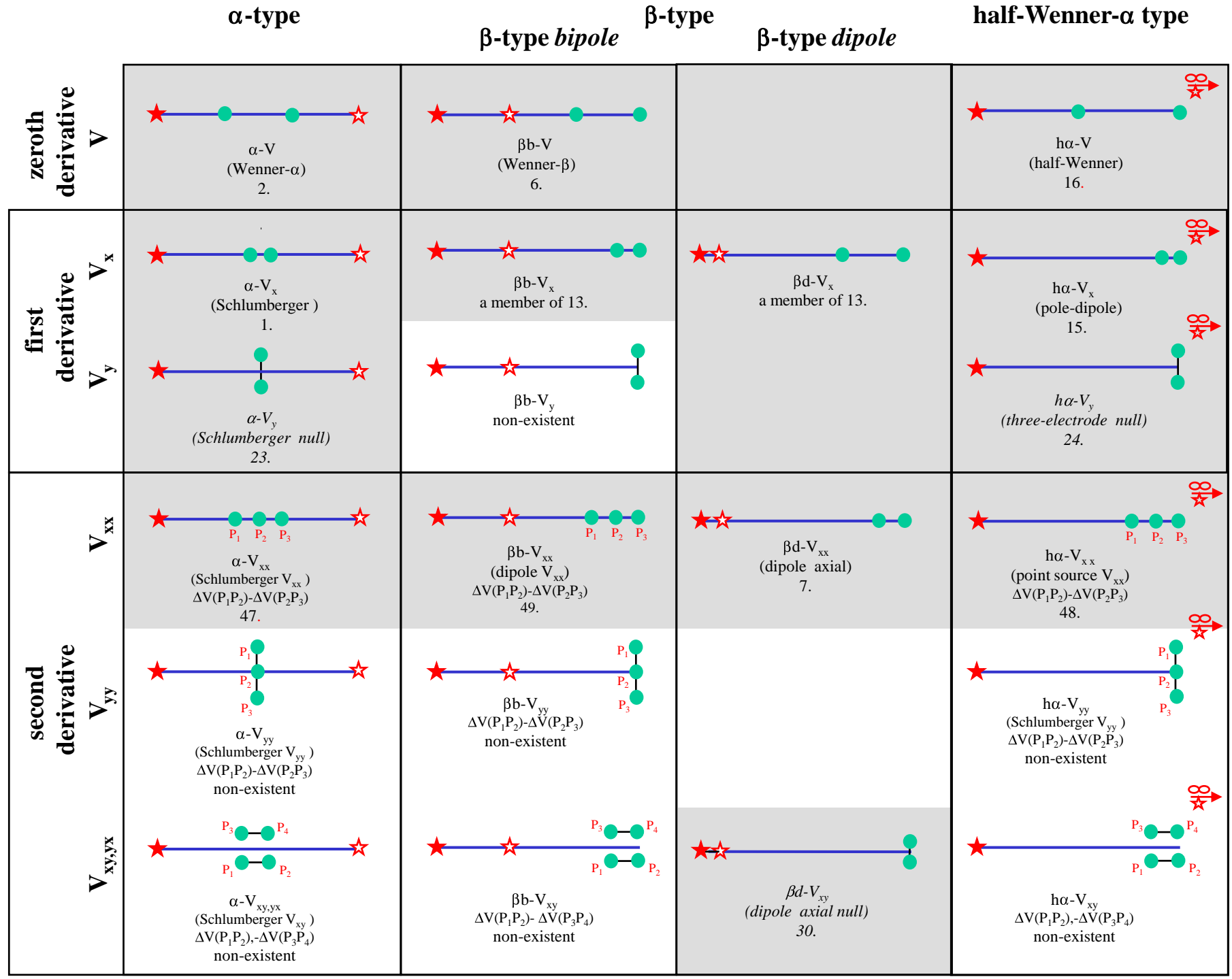

Fig. 7. A systematic collection of arrays to measure various derivatives of the electric potential. The arrays, which have already been applied in field, are indicated with gray backround. Fig. 7 includes the arrays, which represent solution technique 3b ("Derivation") to satisfy the original intention "Measuring resistivity changes in the measuring direction" with the main consideration "Studying inhomogeneity, anisotropy". 


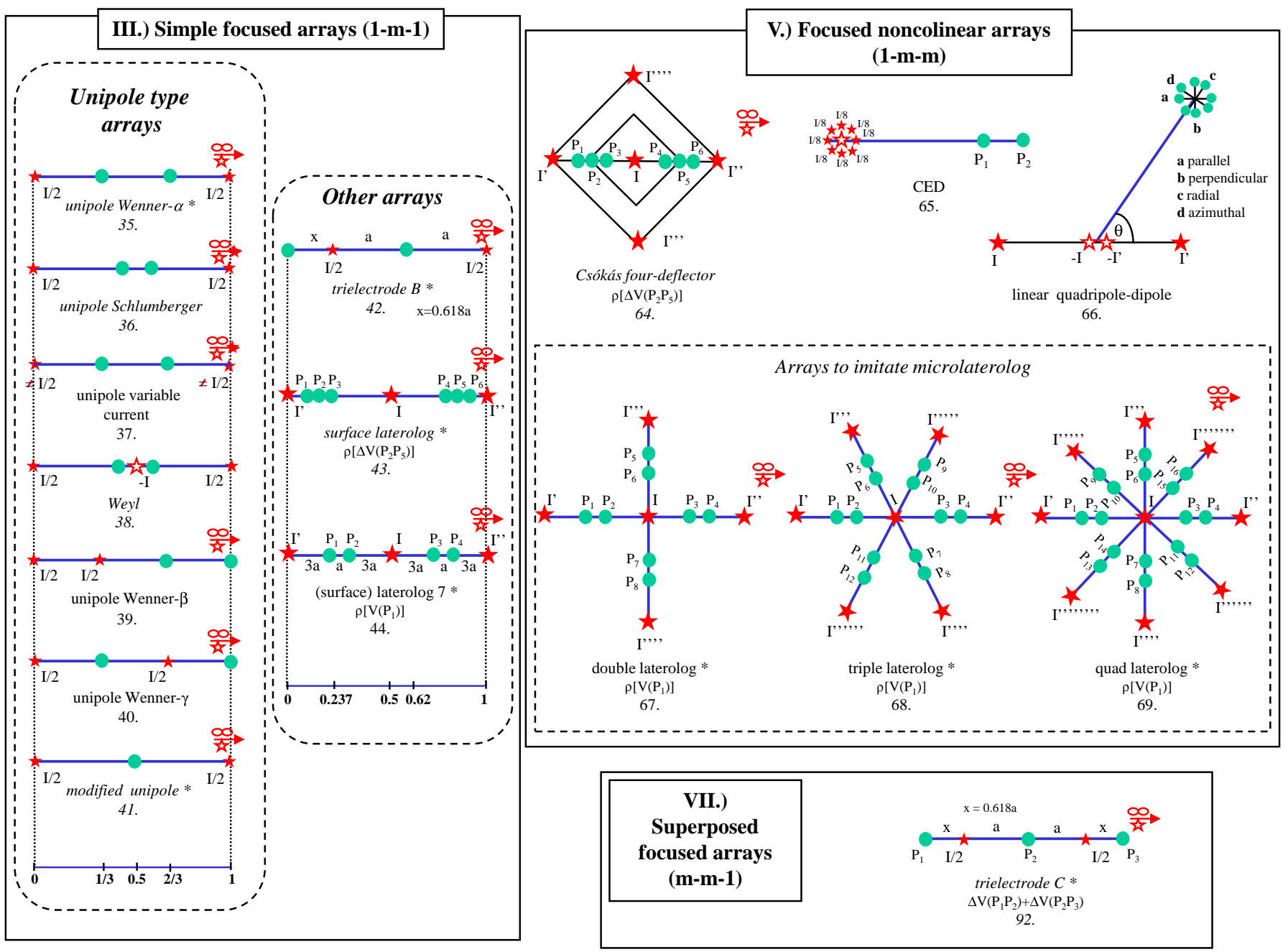

Fig. 8. The 17 electrode arrays, which represent solution technique 4 ("Focused arrays") to satisfy the original intention "Sending the currents deeper" with the main consideration "Enhancement of the depth of investigation".

hypothesis proved false (Szalai et al., 2009). At the same time, the focused arrays seem to have another advantageous feature: namely they - due to the fact that the downward focused currents create electric charge accumulations on horizontal interfaces, which generate a strong electric field at the surface (Szalai and Szarka, 2008b) are more reliable in areal cross-section imaging: e.g. oil spots on the surface of groundwater, and shape of anticlines. In Fig. 8, both the "simple focused arrays" in group III and the "superposed focused" arrays in group VII are linear (although the latter one is quite complicated). From a practical point of view, arrays 35-42 seem to be especially promising; they are all shown in Fig. 8.

\section{Discussion}

Table 1 lists 18 various suggested solution techniques for 11 various original intentions. The number of array solutions for each of the 18 cases is shown, too. The arrays themselves are shown in Figs. 2-8. Of the 168 array solutions, altogether 65 solutions are either not suitable for $2 \mathrm{D}$ multielectrode measurements or have already been implemented into such systems. At the same time, 103 array solutions would be suitable for 2D multielectrode measurements, but have not been used yet. The 103 array solutions mean altogether 61 independent arrays.

It is worth transforming the historical aspect of the previous section to the present needs of 2D multielectrode measurements: increasing the depth of investigation, the vertical and the horizontal resolution power, having better planview imaging, and eliminating the near-surface inhomogeneities.

The nearly forgotten 61 arrays, which have come up to daylight, offer altogether 103 solutions for 2D multielectrode measurements. The potential benefits and the recommended array solutions are summarized as follows:

1. The depth of investigation is increased if either one of the electrodes is put to infinity (as shown in solution technique $1 \mathrm{~b}$ ) or by using a null array (solution technique $2 \mathrm{a}$ ).

2. The vertical resolution power is increased effectively through reducing the distance between the current and the potential electrodes (solution technique 1a).

3. The horizontal resolution power is increased primarily by using the derivative technique (solution technique $3 \mathrm{~b}$ ).

4. A better planview image can be expected from the focusing technique (solution technique 4 ).

5. The measuring area could be kept to a minimum by using asymmetric arrays (solution technique $1 \mathrm{e}$ ).

6. The effect of near-surface inhomogeneities can be removed by using solution technique $2 \mathrm{~b}, 2 \mathrm{c}, 2 \mathrm{~d}$ and $2 \mathrm{e}$.

\section{Conclusions}

We have collected the original intentions, leading to new geoelectric arrays, together with the various solution techniques for 
arrays. Several nearly-forgotten arrays would be worth incorporating into 2D multielectrode measurements.

A joint application of arrays having complementary features, e.g. a combination of arrays having large depth of investigation (but low vertical resolution) with arrays having low depth of investigation (but high vertical resolution), and their joint inversion seems especially promising.

No concrete array selection is provided; an arsenal of arrays is presented with their specific features, which would be beneficial for 2D multielectrode arrays. On this basis, various multielectrode arrays can be designed. The realization of these ideas is beyond the scope of this paper.

\section{Acknowledgments}

Hungarian National Scientific Research Fund (K49604, NI61013), andJános Bolyai Research Scholarship of the Hungarian Academy of Sciences (S. Szalai).

\section{References}

Candansayar, M.E., Bassokur, A.T., 2001. Detecting small-scale targets by the 2D inversion of two-sided three-electrode data: application to an archaeological survey. Geophys. Prospect. 49, 13-25.
Furman, A., Ferré, T.P.A., Warrick, A.W., 2004. Optimization of ERT surveys for monitoring transient hydrological events using perturbation sensitivity and genetic algorithms. Vadose Zone J. 3, 1230-1239.

Mousatov, A., Pervago, E., Shevnin, V., 2002. Anisotropy determination in inhomogeneous media by tensor measurements of the electric field. Proceedings of SEG 72th Annual Meeting in Salt-Lake City, Nevada, USA

Roy, A., Apparao, A., 1971. Depth of investigation in direct current methods. Geophysics 36, 943-959.

Sapuzhak, Ja S., 1967. Higher Derivatives of the Electrical Potential in Geophysical Prospecting. Naukova Dumka, Kiev. (in Russian).

Stummer, P., Maurer, H.R., Green, A.G., 2004. Experimental design: electrical resistivity data sets that provide optimum subsurface information. Geophysics 69, 120-139.

Szalai, S., Szarka, L., 2008a. On the collection and classification of surface geoelectric arrays. Geophys. Prospect. 56, 159-175.

Szalai, S., Szarka, L., 2008b. Parameter sensitivity maps of surface geoelectric arrays II. Nonlinear and focused arrays. Acta Geod. Geophys Hung. 43, 439-447.

Szalai, S., Szarka, L., Prácser, L., Bosch, F., Müller, I., Turberg, P., 2002. Geoelectric mapping of near-surface karstic fractures by using null arrays. Geophysics 67 , 1769-1778.

Szalai, S., Szarka, L., Marquis, G., Sailhac, P., Kaikkonen, P., Lahti, I., 2005. Collinear null arrays in geoelectrics. IAGA WG 1.2 on electromagnetic induction in the earth. Proceedings of the 17th Workshop. http://www.emindia2004.org.

Szalai, S., Novák, A., Szarka, L., 2009. Depth of investigation and vertical resolution of surface geoelectric arrays. J. Environ. Eng. Geophys. 14, 15-23.

Wilkinson, P.B., Meldrum, P.I., Chambers, J.E., Kuras, O., Ogilvy, R.D., 2006. Improved considerations for the automatic selection of optimised sets of electrical resistivity tomography measurement configurations. Geophys. J. Int. 167, 1119-1126.

Xu, B., Noel, M., 1993. On the completeness of data sets with multielectrode systems for electrical resistivity survey. Geophys. Prospect. 41, 791-801. 